Gefässchirurgie 2010 · 15:324-327

DOI 10.1007/s00772-010-0787-9

Online publiziert: 18. August 2010

(c) Springer-Verlag 2010

N. Diehm ${ }^{1} \cdot$ T. Willenberg $^{1} \cdot$ N. Kucher ${ }^{1} \cdot$ F. Dick ${ }^{2} \cdot$ D.-D. Do ${ }^{1} \cdot$ M. Widmer ${ }^{2}$.

J. Schmidli ${ }^{2} \cdot$ I. Baumgartner $^{1}$

${ }^{1}$ Abteilung für Klinische und Interventionelle Angiologie, Schweizer

Herz- und Gefässzentrum, Inselspital, Universität Bern

${ }^{2}$ Abteilung für Gefässchirurgie, Schweizer Herz- und

Gefässzentrum, Inselspital, Universität Bern

\title{
Gefäßmedizin heute
}

\section{Die Berner Sicht}

Die klinische und sozioökonomische Bedeutung der Gefäßmedizin wird in den nächsten Jahren weiter wachsen. Die moderne Behandlung von Gefäßpatienten stellt eine reizvolle und anspruchsvolle Aufgabe dar, die aufgrund der Komplexität und Breite des Spektrums heute in einem interdisziplinären Gefäßzentrum durchgeführt werden sollte.

Die enge interdisziplinäre Kollaboration von Gefäßchirurgen und klinischen und interventionellen Angiologen hat an der Universität Bern eine lange Tradition, die eine konstruktive Arbeit fernab jeglicher standespolitischer Grabenkämpfe zulässt und den Anspruch erhebt, eine optimale und individuelle Behandlung für jeden Patienten zu gewährleisten $[1,12]$. Gegenseitige Achtung und Respekt sind hierbei eine unabdingbare Grundvoraussetzung, ohne die dieses gemeinsame Ziel leicht aus den Augen verloren gehen kann.
Grundlage für eine solche enge Zusammenarbeit war die Schaffung eines organspezifischen Departments Herz und Gefässe im Jahre 1999 am Universitätsklinikum Bern. Hierzu gehören die Kliniken für Kardiologie, Herz- und Gefäßchirurgie sowie Angiologie. Damit war es möglich, organisatorische und administrative Verbesserungen zu realisieren, die dank kurzer Wege eine interdisziplinäre Therapie täglich rund um die Uhr an 365 Tagen im Jahr erlauben.

\section{Alle vaskulären Patienten werden in einer gemeinsamen angiologisch- gefäßchirurgischen Poliklinik betreut}

Als Ausdruck der engen interdisziplinären Zusammenarbeit werden alle vaskulären Patienten in Bern in einer gemeinsamen angiologisch-gefäßchirurgischen Poliklinik betreut ( $\bullet$ Abb. 1). Hierzu gehören

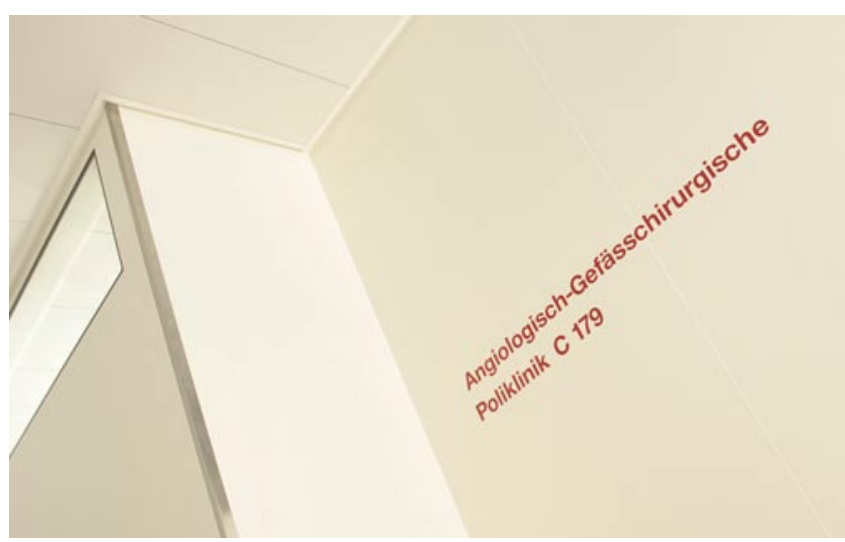

Abb. $1<$ Angiologisch-gefäßchirurgische Poliklinik der Universität Bern neben der klinischen Beurteilung auch die nichtinvasiven Untersuchungen wie die Oszillometrie, die Knöchel-Arm-Index-Bestimmung und die Duplexsonographie sowie die Behandlung der kardiovaskulären Risikofaktoren. Auch wenn der internistisch-angiologische Partner den größeren Routineanteil in der Ambulanz trägt, so bleibt der Entscheid über die Behandlung der Patienten ein gemeinsamer. Ein täglicher Röntgenrapport gewährleistet die konstruktive Diskussion und schafft hierdurch Transparenz und Verständnis für neue Entwicklungen in beiden Disziplinen.

Neben klinisch und wissenschaftlich tätigen angiologischen Ausbildungsassistenten wird die ambulante Betreuung der Patienten personell durch einen Austauschassistenten der Abteilung für Herzund Gefäßchirurgie sowie der Abteilung für Innere Medizin unterstützt. Ein wichtiger neuerer Teil in der Zusammenarbeit zwischen der Gefäßchirurgie und der Angiologie ist eine Ausbildungsrotation von gefäßchirurgischen Fachärzten in die Bereiche Duplexsonographie und Katheterintervention. In Zukunft geplant ist im Gegenzug die Rotation von angiologischen Assistenten in die Gefäßchirurgie. Einerseits um eine bessere Einschätzung für die Indikation von operativen Eingriffen zu bekommen, andererseits um das Verständnis für die im Vorfeld von operativen Eingriffen durchgeführten Duplexsonographien zu optimieren.

Zur Supervision des Poliklinikbetriebs sind jeweils ein Facharzt für Angiologie 
und einer für Gefäßchirurgie verfügbar, um eine direkte interdisziplinäre Besprechung für jeden individuellen Patienten zu ermöglichen.

Alle endovaskulär und offen-chirurgisch behandelten Patienten werden prospektiv in Datenbanken erfasst. So können relevante Details zu Abklärung und Eingriff sowie die klinischen Ergebnisse inklusive Komplikationen und alle im Follow-up relevanten klinischen und apparativen Untersuchungen im Rahmen von Qualitätskontrollen oder Forschungsprojekten abgefragt werden. Eine regelmäßig stattfindende Morbiditäts- und Mortalitätskonferenz dient der systematischen Aufarbeitung und der Qualitätskontrolle.

\section{( Zur Qualitätssicherung finden regelmäßig Morbiditäts- und Mortalitätskonferenzen statt}

Die fachspezifische Weiterbildung der ärztlichen Mitarbeiter erfolgt im Rahmen einer wöchentlich stattfindenden angiologisch-gefäßchirurgischen Fortbildung, die neben einem systematischen Weiterbildungscurriculum die gemeinsame Diskussion verschiedener kardiovaskulärer Themenbereiche und Innovationen aus klinischer und experimenteller Forschung zulässt.

Im klinischen Alltag von Angiologen und Gefäßchirurgen steht die Behandlung von Patienten mit peripher-arterieller Verschlusskrankheit, Erkrankungen des Venensystems, problematischen Dialysefisteln, aortalen und peripheren Aneurysmen sowie Angiodysplasien im Vordergrund. Hierbei ist der klinisch und interventionell tätige Angiologe tagsüber im Rahmen der oben beschriebenen angiologisch-gefäßchirurgischen Poliklinik der primäre Ansprechpartner für Zuweiser und betroffene Patienten. Oftmals stellen diese jedoch eine Herausforderung für Ärzte verschiedenster Disziplinen dar und die gefäßmedizinische Betreuung muss in den Kontext der vorhandenen Komorbiditäten gebettet und Behandlungsempfehlungen in den internistischen Gesamtkontext der individuellen Patienten integriert werden.

Der 24-Stunden-Service wird gemeinsam geleistet. Während der gefäßchirurgische Dienstarzt aufgrund der häu- figer nötigen raschen Verfügbarkeit auch nachts für Notfälle im Hause ist, bietet das angiologische Team neben der täglichen Sprechstunde einen 24-StundenBereitschaftsdienst für Katheterinterventionen und duplexsonographische Diagnostik an.

\section{Peripher-arterielle Verschlusskrankheit (pAVK)}

Mit Zunahme der Prävalenz des Diabetes mellitus und der Lebenserwartung ist eine stetige Zunahme der zu behandelnden Patienten mit pAVK zu sehen [5].

Dreiunddreißig Jahre nach der ersten perkutanen transluminalen Angioplastie (PTA) durch Andreas Grüntzig in Zürich haben sich endovaskuläre Revaskularisationsverfahren deutlich weiterentwickelt [3].

Felix Mahler, ein Pionier auf dem Gebiet der endovaskulären Therapie, hat die mit Grüntzig erarbeitete endovaskuläre Expertise im Jahre 1977 in Bern etabliert und weiterentwickelt.

Unsere Erfahrung hat gezeigt, dass insbesondere die Betreuung von Patienten mit kritischer Extremitätenischämie keine „Einmannshow“ darstellen darf $[4,8]$. Oftmals benötigen diese Patienten die Expertise von Fachleuten verschiedenster Disziplinen, und es muss im Zusammenspiel mit deren Hausärzten ein individuelles Behandlungskonzept für jeden Patienten erarbeitet werden.

Seitens der arteriellen Revaskularisation haben sich heute endovaskuläre Verfahren als primäre Behandlungsmodalität für die meisten Patienten sowohl mit Claudicatio intermittens als auch mit kritischer Extremitätenischämie durchgesetzt [11]. Dies führt einerseits zu einer stetigen Zunahme der Anzahl der durchgeführten Kathetereingriffe, andererseits bedeutet dies aber auch, dass sich das Anforderungsspektrum an Gefäßchirurgen kontinuierlich zu komplexeren Eingriffen (d. h. an Patienten ohne endovaskuläre Behandlungsoption) verschoben hat.

Kathetertechnische Revaskularisationen finden unter der Leitung des jeweiligen interventionell tätigen Kaderarztes der Abteilung für Angiologie in Zusammenarbeit mit Kollegen der interventionellen Radiologie statt. Offenchirurgisch-endovaskuläre Hy-

\section{Hier steht eine Anzeige.}

\author{
Springer
}


Gefässchirurgie 2010 · 15:324-327

DOI 10.1007/s00772-010-0787-9

(c) Springer-Verlag 2010

N. Diehm · T. Willenberg $\cdot$ N. Kucher •

F. Dick - D.-D. Do - M. Widmer - J. Schmidli • I. Baumgartner

Gefäßmedizin heute.

Die Berner Sicht

Zusammenfassung

Die moderne Behandlung von Gefäßpatienten stellt eine reizvolle und anspruchsvolle Aufgabe dar, welche aufgrund der Komplexität und der Breite des Spektrums heute in interdisziplinären Gefäßzentren durchgeführt werden sollte. Die enge Zusammenarbeit zwischen Gefäßchirurgen und Angiologen hat an der Universität Bern eine lange Tradition. Der vorliegende Artikel legt die grundlegende Philosophie unserer gefäßchirurgisch-angiologischen Freundschaft und Zusammenarbeit dar und schildert deren Umsetzung im klinischen Alltag.

\section{Schlüsselwörter}

Gefäßzentrum · Peripher-arterielle Verschlusskrankheit · Aortenaneurysma · Venenerkrankungen $\cdot$ Angiodysplasie

\section{Up-to-date management of vascular patients. The Bern perspective}

\begin{abstract}
Up-to-date management of patients with vascular disorders is a challenging yet very interesting undertaking that nowadays, because of the complexity and broad spectrum of disease, should be addressed in interdisciplinary vascular centers. The close collaboration of vascular surgeons and clinical and interventional angiologists has a long-standing tradition at the University Hospital of Bern. This article outlines the philosophy of the partnership between vascular surgeons and angiologists and highlights typical clinical examples of this fruitful collaboration.
\end{abstract}

Keywords

Vascular center - Peripheral arterial disease . Aortic aneurysm - Venous disease - Angiodysplasia brideingriffe werden gemeinsam im Operationssaal durch Gefäßchirurgen und Angiologen durchgeführt. Somit ergänzt die endovaskuläre Expertise optimal diejenige der chirurgisch tätigen Kollegen. Weiterhin gestattet dies den interventionell tätigen Angiologen einen direkten Einblick in den Situs der zu behandelnden vaskulären Pathologie, die sich im Katheterlabor nur angiographisch darstellt.

Als Teil dieses Konzeptes rotiert jeweils ein sich in gefäßchirurgischer Facharztausbildung befindlicher Kollege unter Anleitung eines erfahrenen interventionellen Angiologen für 4 Monate ins Katheterlabor. Dies ermöglicht eine endovaskuläre Grundausbildung für Gefäßchirurgen und erleichtert die zukünftige Kommunikation und Zusammenarbeit aus unserer Erfahrung erheblich.

\section{Aneurysmen}

Die moderne Behandlung des Patienten mit Bauchaortenaneurysma (BAA) umfasst weitaus mehr als die chirurgische oder endovaskuläre Sanierung des Aneurysmas.

Im Rahmen unserer angiologisch-gefäßchirurgischen Poliklinik unterziehen wir alle männlichen Patienten $>65$ Jahren einem sonographischen Screening der Bauchschlagader. Die Anzahl unmittelbar operationsbedürftiger BAAs, die sich im Rahmen solcher Screenings finden lassen, ist klein. Jedoch weisen BAA-Patienten neben der Rupturgefahr auch deutlich erhöhte kardiovaskuläre Mortalitätsraten auf, sodass der Befund auch eines kleinen BAA hinsichtlich der kardiovaskulären Prognose als KHK-Risiko-Äquivalent gilt [9]. Somit ergibt sich für Patienten mit kleinen BAAs neben der Chance der adäquaten Festlegung der sonographischen Verlaufskontrollen des Aneurysmas (mit dem Ziel die Indikationsgrenze für ein aktives Vorgehen nicht zu verpassen) [2] auch die Möglichkeit der optimalen medikamentösen Versorgung zur Sekundärprävention $[6,7]$.

Besteht bei BAA-Patienten die Indikation zu einem aktiven offen-chirurgischen oder endovaskulären Vorgehen, so wird diese auf der angiologisch-gefäßchirurgischen Poliklinik gemeinsam mit dem Patienten interdisziplinär besprochen und unter Einbezug des Hausarztes im medizinischen Gesamtkontext des jeweiligen Patienten festgelegt. Zwischen einem Viertel und einem Drittel aller Patienten eignen sich an unserer Klinik morphologisch für ein endovaskuläres Vorgehen und werden entsprechend behandelt. Die übrigen Patienten werden offen operiert. Obwohl sich in unserem Tertiärzentrum die Patienten mit komplexerer Anatomie der Landezonen sammeln, entspricht dieser Schnitt ungefähr den Schätzungen der europäischen Sammelstatistik des EVEM-Panels und zeigt, dass die interdisziplinäre Beurteilung zu einem repräsentativen Einsatz der Behandlungsoptionen führt.

Grundsätzlich werden endovaskuläre BAA-Eingriffe gemeinsam durch ein Team von Gefäßchirurgen und interventionellen Angiologen durchgeführt. Hierbei ergänzen sich chirurgische und endovaskuläre Erfahrungen synergistisch.

\section{(-) Die chirurgischen und endovaskulären Erfahrungen ergänzen sich synergistisch}

Aufgrund unserer Erfahrungen führen wir bei allen BAA-Patienten eine weiterführende duplexsonographische Untersuchung der Becken- und Beinschlagadern durch (peripheres Aneurysma-Screening), weil sich hier nicht selten weitere Manifestationen der dilatativen und/oder obstruierenden Arteriopathie finden [13].

Die Behandlung des Poplitealarterienaneurysmas erfolgt heute in unserem Zentrum bei einem Großteil der Patienten nach wie vor offen-chirurgisch. In morphologisch geeigneten Einzelfällen kommt aber auch hier eine endovaskuläre Behandlung durch Implantation eines gecoverten Stents in Betracht.

Die Nachsorge und Verlaufskontrolle aller Aneurysmapatienten wird auf der angiologisch-gefäßchirurgischen Poliklinik durchgeführt [12].

\section{Venenerkrankungen}

Der Bereich der Phlebologie als Teilgebiet der Angiologie integriert unter deren Obhut eine interdisziplinäre Zusammenarbeit mit der Gefäßchirurgie und der Dermatologie. Die inzwischen mit mehreren gleichwertigen Optionen zu behandelnde Vari- 
kose, sowie die oft komplexen Eingriffe im Bereich des tiefen Venensystems sind heute nur über diesen interdisziplinären Weg mit nachhaltiger Qualität möglich. Beispielgebend sei der Hybrideingriff einer frischen deszendierenden Beckenvenenthrombose erwähnt, bei welcher die chirurgische Thrombektomie durch die interventionellen Beseitigung einer Beckenvenenobstruktion mittels Stenting komplettiert wird [9]. Diagnostik und Nachsorge der Patienten erfolgt primär durch den Angiologen, der hierbei nicht selten auch seine internistische Kompetenz einbringen muss, beispielsweise in der Entscheidung über die Dauer der Antikoagulation. Im Bereich des oberflächlichen Venensystems hat sich bei der Therapie der Varikose die ultraschallgesteuerte Schaumsklerotherapie als sehr effektive Behandlungsoption neben der klassischen Chirurgie und den modernen endovenösen Verfahren bewährt.

Die Behandlung der akuten Lungenembolie wird nach wie vor kontrovers diskutiert. Durch die rasche Diagnosestellung mittels Computertomographie und Echokardiographie kann eine Reperfusionstherapie (Thrombolyse, kathetertechnische Thrombektomie, chirurgische Embolektomie) bei Patienten mit massiver Lungenembolie und arterieller Hypotonie lebensrettend sein. Die systemische Thrombolyse kann bei hämodynamisch instabilen $\mathrm{Pa}$ tienten ohne Risikofaktoren für Blutungen mit Erfolg eingesetzt werden. Patienten mit massiver Lungenembolie und erhöhtem Blutungsrisiko, wie z. B. die massive postoperative Lungenembolie, profitieren von einer notfallmäßig durchgeführten chirurgischen Embolektomie oder einer kathetertechnischen Intervention mit Fragmentation und Aspiration der pulmonalen Embolien. In jedem Falle sollte die Indikationsstellung im Konsens mehrerer Fachdisziplinen erfolgen, insbesondere wenn keine unmittelbar lebensbedrohliche Situation für den Patienten vorliegt.

In ähnlicher Weise erfolgt die Betreuung von Patienten mit Dialysefisteln. Nach Zuweisung durch unsere Kollegen der Nephrologie werden die Patienten im Duplexlabor primär angiologisch beurteilt. Die Frage, ob ein Fistelproblem primär endovaskulär oder offenchirurgisch behandelt wird, wird interdisziplinär zwischen dem zuständigen angiologischen und gefäßchir- urgischen Facharzt unter Einbezug des zuweisenden Nephrologen diskutiert.

Vaskuläre Malformationen gehören zu den Raritäten der Gefäßmedizin. Die betroffenen Patienten sollten in jedem Falle einem auf diesem Gebiet erfahrenem Zentrum zugewiesen werden [14]. In Bern besteht bereits seit vielen Jahren ein interdisziplinäres Board, welches unter Obhut der plastischen Chirurgie und der Angiologie gemeinsam mit den weiteren Beteiligten verschiedenster Fachdisziplinen komplexe vaskuläre Malformationen diskutiert und betreut [14]. Während bei den sog. arteriovenösen High-flow-Läsionen die endovaskuläre Embolisation in den Vordergrund gerückt ist, lassen sich die meisten venösen Malformationen mittels Sklerotherapie erfolgreich behandeln [14]. Lymphatische Malformationen hingegen können mit der Applikation von OK 432 (Picibanil) angegangen werden [14]. Bereits bei der umfangreichen Diagnostik dieser Gefäßmalformationen ist eine interdisziplinäre Expertise gefragt, um die für das therapeutische Konzept entscheidenden Fragen zu beantworten. Diese kann nur in einem Team wachsen, welches wie im Berner Modell über Jahre zusammenarbeitet.

\section{Fazit für die Praxis}

Das oben dargelegt Konzept hat sich am Inselspital in Bern über Jahre zum Erfolgsmodell für alle Beteiligten entwickelt. Die vielerorts immer noch gehegte Befürchtung, dass interventionell tätige Angiologen durch endovaskuläre Eingriffe das Volumen gefäßchirurgischer Eingriffe dramatisch reduzieren, können wir nicht bestätigen. Vielmehr stellt sich zunehmend heraus, dass offen-chirurgische und endovaskuläre Behandlungskonzepte komplementäre und nicht konkurrierende Methoden sind, die sich, richtig angewendet, optimal ergänzen und die Behandelbarkeit unserer gemeinsamen Patienten entscheidend verbessern und erweitern. Diese lokale Tatsache findet auch zunehmend global in internationalen Guidelines ihren Niederschlag.

Wie in jeder Partnerschaft erfordert diese Art der interdisziplinären Zusammenarbeit selbstredend eine aufrichtige Kommunikation und Kompromissbereitschaft aller Beteiligten.

\section{Korrespondenzadresse}

\section{PD Dr. N. Diehm}

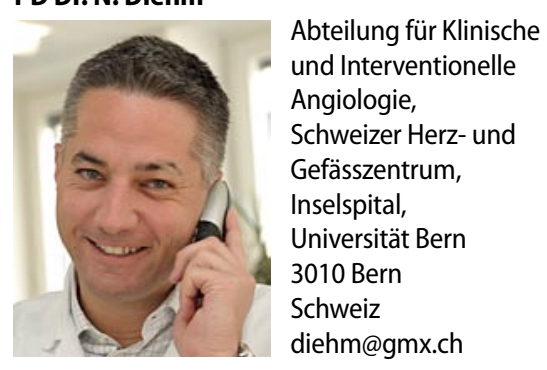

Interessenkonflikt. Der korrespondierende Autor gibt an, dass kein Interessenkonflikt besteht.

\section{Literatur}

1. Baumgartner I, Schainfeld R, Graziani L (2005) Management of peripheral vascular disease. Annu Rev Med 249-272

2. Brady AR, Thompson SG, Fowkes FG et al (2004) Abdominal aortic aneurysm expansion: risk factors and time intervals for surveillance. Circulation 110:16-21

3. Cremonesi A, Castriota F, Diehm N et al (2009) Peripheral arterial disease. In: Camm AJ, Luescher T, Serruys PW (eds) The ESC textbook of cardiovascular medicine. Blackwell Scientific, Okford, UK

4. Dick F, Diehm N, Galimanis A et al (2007) Surgical or endovascular revascularization in patients with critical limb ischemia: influence of diabetes mellitus on clinical outcome. J Vasc Surg 45:751-761

5. Diehm C, Schuster A, Allenberg JR et al (2004) High prevalence of peripheral arterial disease and co-morbidity in 6880 primary care patients: crosssectional study. Atherosclerosis 172:95-105

6. Diehm N, Baumgartner I (2008) Neue Erkenntnisse in der medikamentösen Behandlung infrarenaler Aortenaneurysmen. J Gefässmed 5:11-14

7. Diehm N, Dick F, Schaffner T et al (2007) Novel insight into the pathobiology of abdominal aortic aneurysm and potential future treatment concepts. Prog Cardiovasc Dis 50:209-217

8. Diehm N, Silvestro A, Baumgartner I et al (2009) Chronic critical limb ischemia: European experiences. J Cardiovasc Surg (Torino) 50:647-653

9. Expert Panel on Detection, Evaluation, and Treatment of High Blood Cholesterol in Adults (2001) Executive Summary of the Third Report of the National Cholesterol Education Program (NCEP) expert panel on detection, evaluation and treatment of high blood cholesterol in adults (Adult Treatment Panel III). JAMA 285:2486-2497

10. Husmann MJ, Heller G, Kalka C et al (2007) Stenting of common iliac vein obstructions combined with regional thrombolysis and thrombectomy in acute deep vein thrombosis. Eur JVasc Endovasc Surg 34:87-91

11. Norgren L, Hiatt WR, Dormandy JA et al (2007) Inter-society consensus for the management of peripheral arterial disease (TASC II). J Vasc Surg 45:S5S67

12. Widmer M, Dick F, Schmidli J (2009) Gefässchirurgie in der Praxis - die Berner Perspektive. Hans Huber, Bern

13. Widmer MK, Blatter S, Schmidli J et al (2008) Generalized dilating diathesis in patients with popliteal arterial aneurysm. Vasa 37:157-163

14. Willenberg T, Baumgartner I (2008) Vascular birthmarks. VASA 37:5-17 